\title{
Yeast membranes and cell wall: from basics to applications
}

\author{
Ivan Hapala • Peter Griač • Jozef Nosek • \\ Hana Sychrová $\cdot$ Ľubomír Tomáška
}

Received: 3 September 2013/Revised: 11 September 2013/Accepted: 12 September 2013/Published online: 22 September 2013

(C) Springer-Verlag Berlin Heidelberg 2013

The surfaces of cells and organelles are crucial territories whose proper structure and function are essential for all cellular activities. In Saccharomyces cerevisiae, the cell envelope alone (plasma membrane and cell wall) occupies about $15 \%$ of the total cell volume (Feldmann 2010). After adding in the membranes of the mitochondria, nuclei, vacuoles, peroxisomes, endoplasmic reticulum and Golgi apparatus as well as a monolayer covering the surface of lipid particles, there is very little room left for the cytosol or organellar matrices. The cell and organellar surfaces sense the environment, relay this information to the interior via signal transduction pathways, mediate (or prevent) the entry of chemicals, excrete toxic substances, enable an uneven distribution of ions thus generating ion and $\mathrm{pH}$ gradients that are the driving forces for essential cell processes. Yeast cell surfaces also facilitate the communication of a cell with its neighbors, and, in a yeast pathogen,

Communicated by S. Hohmann.

Special Issue: Yeast membranes and cell wall: From basics to applications

\section{Hapala · P. Griač}

Institute of Animal Biochemistry and Genetics, Slovak Academy of Sciences, Moyzesova 61, 90028 Ivanka pri Dunaji, Slovak Republic

\section{J. Nosek · Ľ. Tomáška $(\bowtie)$}

Departments of Biochemistry and Genetics, Faculty of Natural

Sciences, Comenius University, Mlynská dolina, 84215

Bratislava, Slovak Republic

e-mail: tomaska@fns.uniba.sk

\section{H. Sychrová}

Institute of Physiology, Academy of Sciences of the Czech

Republic, v.v.i., Vídeňská 1083, 14220 Prague 4, Czech

Republic interact with the host immune system. These and other roles of the cell and organelle surfaces are widely recognized, yet far from fully understood.

Current knowledge in this field has been built on pioneering studies, of which a substantial number were conducted by Czech and Slovak scientists in the 1960s. Among others, Oldřich Nečas and his colleagues in Brno established a world-class school of cell wall biogenesis (Nečas 1961), Eva Streiblová (1968) and her students in Prague perfected various microscopic techniques and opened the yeast cell up to direct observation of its membranous structures, and Ladislav Kováč and his co-workers in Bratislava employed yeast mutants to elucidate processes involved in mitochondrial bioenergetics and biogenesis (Kováč 1974).

This long tradition of research on the yeast cell wall and organelles in the former Czechoslovakia was reflected by the fact that for its 30th anniversary, the International Specialized Symposium on Yeast (ISSY) returned to the country where the 1st ISSY was held in Smolenice castle in 1971 and was dedicated to the yeast cell surface and organelles. The conference (http://www.issy2013.org) was organized by the International Commission on Yeasts (ICY; http://icyeast.org) and the Yeast Commission of the Czechoslovak Society for Microbiology (http://www.chem. sk/yeast/indexen.htm). The meeting was held in June 18-23, 2013 in Stará Lesná in the High Tatras. The core of these crystalline granitoids was formed from solidified magma in the Paleozoic Era (cca 335 million years ago; Poller and Todt 2000). This event took place $400-800$ million years after Ascomycota diverged from Basidiomycota and the first yeast-like organisms appeared on Earth (Hedges et al. 2004; Scannell et al. 2007). Since then various geological processes have resulted in a formation of a wide variety of beautiful alpine peaks, valleys, lakes 
and creeks, while biological evolution has produced a vast diversity of yeast species possessing many fascinating features. Saccharomyces cerevisiae as well as a number of non-Saccharomyces species are not only excellent model organisms for fundamental research that continues to make significant contributions to our understanding of human biology, but are prominent microorganisms in the production of food and beverages, as well as in the pharmaceutical and biotechnological industry. At the same time, many yeasts are significant human pathogens.

The diversity of yeasts was reflected by the wide variety of nearly 50 talks and about the same number of posters (for a complete list, see ISSY2013 Proceedings). In the introductory EMBO lecture, Thomas Langer (University of Cologne) described the role of Ups1 and Mdm35 proteins in shuttling phosphatidic acid between mitochondrial membranes and thus regulating the biosynthesis of cardiolipin and phosphatidylethanolamine in mitochondria. In the Dynamics of Membranous Organelles in Yeast session, Frank Madeo (University of Graz) presented evidence for the ability of spermidine to extend chronological life span not only in yeast, but also in Caenorhabditis elegans and mice, possibly via the autophagy pathway. Chris Meisinger (University of Freiburg) employed a proteomic approach to identify phosphorylated mitochondrial proteins and concentrated on the role of phosphorylation in the regulation of the TOM complex involved in protein import across the outer mitochondrial membrane. Franz Lang (University of Montreal) presented provocative data indicating the existence of large super complexes of mitochondrial proteins composed of enzymes of whole (or part of) metabolic pathways and frequently containing unexpected proteins from non-mitochondrial compartments.

In his keynote lecture, André Goffeau (Université Catholique de Louvain) charmingly recapitulated the path from the first pleiotropic drug resistance mutant (Rank and Bech-Hansen 1973) to the intricate network of pumps and transcription factors mediating the response of a yeast cell to xenobiotics.

The importance of non-conventional yeasts for industrial applications was underlined in two biotechnological sessions (Yeast in Biotechnology I and II). Quinn Zhu (DuPont) demonstrated the power of metabolically engineered cells of Yarrowia lipolytica for the production of polyunsaturated fatty acids and their application in the fish farming industry, and Diethard Mattanovich (University of Natural Resources and Life Sciences, Vienna) described the role of redox state in the secretory pathway and organelle dynamics for the improvement of industrial strains of Pichia pastoris.

The Membrane Lipid Homeostasis session was mainly focused on two topics. The first-neutral lipid homeostasis-was introduced by Guenther Daum (University of
Technology Graz) in his talk on regulatory interactions between the enzymes catalyzing synthesis and the degradation of neutral lipids. He demonstrated that Tg13p, the major triacylglycerol lipase in yeast, also possesses acyltransferase activity and that only the protein found in lipid droplets exhibits enzymatic activity. In the second topic related to the role of lipids in antifungal activity and resistance, Rajendra Prasad (Jawaharlal Nehru University) reported the unique response of the cellular lipidome in various Candida albicans isolates resistant to azole antimycotics and the links between the altered lipid homeostasis in these isolates to the functions of mitochondria, plasma membrane and cell walls.

In the Cell Wall Dynamics session, Vladimir Mrša (University of Zagreb, Croatia) summarized the functions of proteins involved in the biogenesis of cell wall architecture and Laura Popolo (Universita degli Studi di Milano) highlighted the action of glucan-remodeling enzymes in the morphogenesis and developmental transitions of pathogenic yeasts.

The program of the 30th ISSY included a special Yeast Microscopy Workshop: Mission Possible aimed at introducing new powerful imaging methodologies to the yeast community. Both invited talks were focused primarily on the organization of the yeast plasma membrane. Roland Wedlich-Söldner (Max Planck Institute of Biochemistry) demonstrated in his talk that the plasma membrane in yeast is laterally organized into a patchwork of functionally distinct domains. In the second talk, Jan Malinsky (Institute of Experimental Medicine ASCR) summarized the results on the structure of the Membrane Compartment of Can1 (MCC) and associated cytoplasmic protein complex (eisosome) that were obtained by a combination of fluorescence and electron microscopy.

In the session focused on fungal pathogens (Biomedical Aspects: Antifungals, Resistance and Biofilms), Patrick van Dijck (Katholieke Universiteit Leuven) introduced new tools for studying biofilm formation directly in animal models, and Alessandro Fiori (Katholieke Universiteit Leuven) demonstrated the role of iron-sulfur cluster biogenesis in the fluconazole tolerance of $C$. albicans and $S$. cerevisiae.

In the last session, Membrane Transporters, Johan Thevelein (Katholieke Universiteit Leuven, Leuven, Belgium) presented an overview of the role of transporters in signaling, and Lynne Yenush (Polytechnical University of Valencia, Spain) described the necessity of efficient intracellular protein trafficking for ion and nutrient homeostasis. The other talks in this session dealt with the characterization of various yeast transporters.

To share exciting developments in the field with a wider audience, seven groups prepared review articles that constitute this special issue of Current Genetics. Lynne 
Yenush and her colleagues summarize their views on the endocytic regulation of alkali metal transport proteins in mammals, yeast and plants (Mulet et al. 2013). Lotte Mathé and Patrick Van Dijck recapitulate recent insights into $C$. albicans biofilm resistance mechanisms (Mathé and Van Dijck 2013). Francois et al. (2013) (Université Toulouse) describe the use of atomic force microscopy (AFM) for the characterization of cell wall properties and response to stress. Rajendra Prasad and Ashutosh Singh illustrate the role of lipids in the multidrug resistance of Candida species (Prasad and Singh 2013). Renata Teparić and Vladimir Mrša (2013) provide a list and characterization in the biogenesis of the yeast cell wall. Johan Thevelein and colleagues depict the concept of transceptors (transporters fulfilling the role of nutrient sensors) and their role in the cAMP-dependent signaling pathway. In addition they summarize data supporting the view of transporters as dynamic proteins undergoing multiple substrate-induced conformational changes, which in concert with other regulators trigger specific downstream events (Schothorst et al. 2013). Finally, Sepp Kohlwein and colleagues (University of Graz) present current view on the regulatory and structural components driving lipid droplet formation and their physiological and pathophysiological roles in lipid homeostasis (Radulovic et al. 2013). We hope that you will enjoy reading these fascinating stories just as much as we enjoyed the ISSY meeting in Stará Lesná.

\section{References}

Feldmann H (2010) Yeast: molecular and cell biology. WileyBlackwell, Weinheim

Francois JM, Formosa C, Schiavone M, Pillet F, Martin-Yken M, Dague E (2013) Use of atomic force microscopy (AFM) to explore cell wall properties and response to stress in the yeast Saccharomyces cerevisiae. Curr Genet (this issue)

Hedges SB, Blair JE, Venturi ML, Shoe JL (2004) A molecular timescale of eukaryote evolution and the rise of complex multicellular life. BMC Evol Biol 4:2

ISSY2013 (2013) In: Proceedings of the 30th International Specialised Symposium on Yeast: Cell Surface and Organelles in Yeasts: from Basics to Applications. Stará Lesná, Slovakia, June 18-22 [ISSN 1336-4839]

Kováč L (1974) Biochemical mutants: an approach to mitochondrial energy coupling. Biochim Biophys Acta 346:101-135

Mathé L, Van Dijck P (2013) Recent insights into Candida albicans biofilm resistance mechanisms. Curr Genet (this issue)

Mulet JM, Llopis-Torregrosa V, Primo C, Marqués MC, Yenush L (2013) Endocytic regulation of alkali metal transport proteins in mammals, yeast and plants. Curr Genet (this issue)

Nečas O (1961) Physical conditions as important factors for the regeneration of naked yeast protoplasts. Nature 192:580-581

Poller U, Todt W (2000) U-Pb single zircon data of granitoids from the High Tatra Mountains (Slovakia): implications for the geodynamic evolution. Trans Royal Soc Edinburgh Earth Sci 91:235-243

Prasad R, Singh A (2013) Candida lipids and their role in multidrug resistance. Curr Genet (this issue)

Radulovic M, Knittelfelder O, Cristobal-Sarramian A, Kolb D, Wolinski H, Kohlwein SD (2013) The emergence of lipid droplets in yeast: current status and experimental approaches. Curr Genet (this issue)

Rank GH, Bech-Hansen NT (1973) Single nuclear gene inherited cross resistance and collateral sensitivity to 17 inhibitors of mitochondrial function in $S$. cerevisiae. Mol Gen Genet 126:93-102

Scannell DR, Butler G, Wolfe KH (2007) Yeast genome evolution: the origin of the species. Yeast 24:929-942

Schothorst J, Kankipati H, Conrad M, Samyn DR, Van Zeebroeck G, Popova Y, Rubio-Texeira M, Persson BL, Thevelein JM (2013) Yeast nutrient transceptors provide novel insight in the functionality of membrane transporters. Curr Genet (this issue)

Streiblová E (1968) Surface structure of yeast protoplasts. J Bacteriol 95:700-707

Teparić R, Mrša V (2013) Proteins involved in building, maintaining and remodeling of yeast cell walls. Curr Genet (this issue) 